\title{
NA.SA-TM-106.282
}

NASA Technical Memorandum 106282

ICOMP-93-27

\section{On Curve Veering and Flutter of Rotating Blades}

Daré Afolabi

Institute for Computational Mechanics in Propulsion

Lewis Research Center

Cleveland, Ohio

and Purdue University

Indianapolis, Indiana

and

Oral Mehmed

Lewis Research Center

Cleveland, Ohio

August 1993

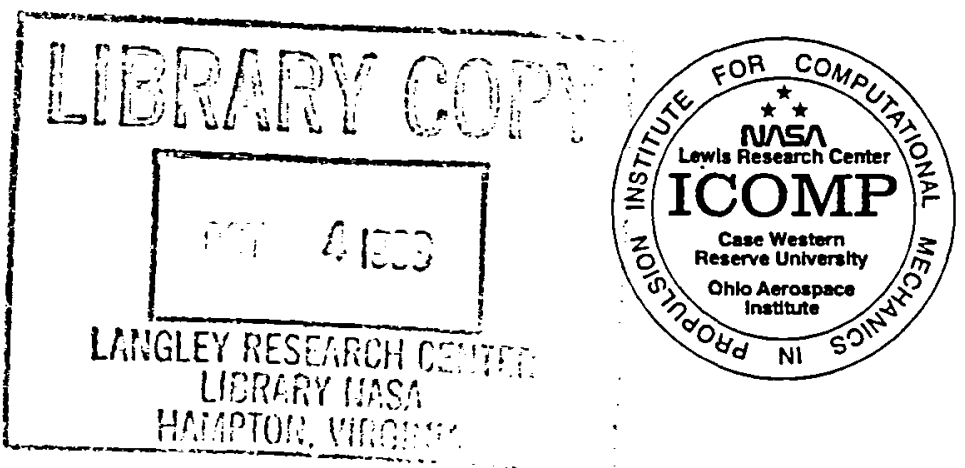


, . 


\title{
ON CURVE VEERING \\ AND FLUTTER OF ROTATING BLADES
}

\author{
DARÉ AFOLABI \\ Department of Mechanical Engineering ${ }^{1}$ \\ Purdue University, Indianapolis, Indiana. \\ and \\ Institute for Computational Mechanics in Propulsion \\ NASA Lewis Research Center, Cleveland, Ohio. \\ Oral Mehmed \\ NASA Lewis Research Center, Cleveland, Ohio.
}

\begin{abstract}
The eigenvalues of rotating blades usually change with rotation speed according to the Stodola-Southwell criterion. Under certain circumstances, the loci of eigenvalues belonging to two distinct modes of vibration approach each other very closely, and it may appear as if the loci cross each other. However, our study indicates that the observable frequency loci of an undamped rotating blade do not cross, but must either repel each other (leading to "curve veering"), or attract each other (leading to "frequency coalescence"). Our results are reached by using standard arguments from algebraic geometry-the theory of algebraic curves and catastrophe theory. We conclude that it is important to resolve an apparent crossing of eigenvalue loci into either a frequency coalescence or a curve veering, because frequency coalescence is dangerous since it leads to flutter, whereas curve veering does not precipitate flutter and is, therefore, harmless with respect to elastic stability.
\end{abstract}

\footnotetext{
${ }^{1}$ On leave. Temporary address: Room 3-360, Department of Mechanical Engineering, MIT, Cambridge, MA 02139.
} 


\section{INTRODUCTION}

Rotating blades are commonly encountered in a variety of mechanical and aeronautical structures such as turbines, compressors, propfans, bladed-disk assemblies, and helicopters. It is well known that such blades may flutter when a sufficiently high critical speed is reached or exceeded. In this paper, the term 'flutter' is used in a generalized sense as frequently done in applied mechanics to mean all kinds of self-excited oscillatory phenomena, with an exponential growth in time. In this sense, flutter may occur without the participation of aerodynamic forces, as in systems with 'follower force'. Also, there are basically two types of flutter-'single mode flutter' and 'coalescence flutter'. This study is mainly about coalescence flutter.

The problem of flutter in general is of vital importance not only in the case of rotating blades, but also with respect to various engineering structures and components in aeronautics. At the Lewis Research Center of the National Aeronautics and Space Administration, a variety of theoretical and experimental investigations of flutter is currently in progress. In this paper, we present a summary of a theoretical investigation of the flutter of rotating blades using mathematical techniques from algebraic geometry, i.e. catastrophe theory (Arnol'd, 1983), and the theory of real algebraic curves (Brieskorn, 1986).

An important conclusion from our investigation is the following. Frequency loci belonging to two distinct modes of vibration will intersect each other only when the coupling between the two modes is assumed to be completely absent. On the other hand, the presence of a small but non-vanishing amount of coupling will cause the two loci to either repel each other (leading to curve veering), or attract each other (leading to frequency coalescence). Frequency crossing is illustrated in Fig. 1 (a), while curve veering and frequency coalescence are depicted, respectively, in Figs. 1 (b) and 1 (c). Curve veering is commonly called "avoided crossing", especially in the physics literature. The term "curve veering" seems to have been introduced to the engineering literature by Leissa (1974) in his study of the eigenvalues of rectangular plates, while frequency coalescence is well known in aeroelasticity as "coupled mode flutter", Bisplinghoff \& Ashley (1962).

The procedure used in arriving at our main result originates from the qualitative method of mathematical models. The qualitative method was initiated by Poincaré (1892) for the study of differential equations. It was developed further by Andronov \& Vitt (1966), who introduced the concept of "structural stability". The structural stability idea was further developed by Thom (1972) into catastrophe theory — "a general theory of models" for applications in the physical, social and biological sciences.

There exist several studies in the literature on rotating blades where a crossing of eigenvalue loci, as in Fig. 2 (a), has been reported. For examples, one may cite the studies of MacBain (1975, his Fig. 10), Chen \&. Dugundji (1984, Fig. 5), Ramamurti \& Kielb (1984,

Figs. 4 and 10), among others. The principal reason why crossings of eigenvalue loci have 
been published by many investigators is that the small but inevitable amount of "coupling" between two interacting modes (such as bending and torsion) is usually ignored by such investigators.

In reality, as distinct from theory, a small amount of coupling is unavoidable when two eigenvalue loci approach each other very closely; see Section 2.3. This coupling could be "conservative" or "non-conservative" in the terminology of Crandall and Mroszczyk (1988). Conservative coupling leads to curve veering, whereas non conservative coupling leads to frequency coalescence. The role of coupling on curve veering has been discussed by Perkins \& Mote, Jr. (1988). However, the influence of coupling on the topology of eigenvalue loci is, in general, usually overlooked or considered to be inconsequential by most investigators, in their theoretical models of flutter of rotating blades. Consequently, when the results of a large number of theoretical studies are examined, one finds that two or more eigenvalue loci do intersect one another in such studies. Such results are, however, in serious qualitative error, and have particular significance in propulsion systems in which the blading has a low mass ratio.

\section{Eigenloci Crossing, Veering or Coalescence}

In this section, we carry out a background discussion on the phenomena of frequency crossing, curve veering, and eigenvalue coalescence. Our prototype element in this discussion is the locus of an eigenvalue of a rotating blade as a function of the rotation speed.

\subsection{Variation of Eigenvalues with Rotation Speed}

It is now well known, due to the works of Stodola (1914), Lamb \& Southwell (1921) and Southwell (1922), that the natural frequencies of a rotating blade or uniform disk vary approximately as quadratic functions of the rotation speed, i.e.,

$$
\omega_{m}^{2}(\Omega)=\omega_{m}^{2}(0) \pm s_{m} \Omega^{2}
$$

where $m$ denotes a mode of vibration, $\Omega$ is the rotation speed, $\omega_{m}(\Omega)$ is the natural frequency at that speed, $\omega_{m}(0)$ is the bench natural frequency, measured when the rotation speed is zero, and $s_{m}>0$ is a Stodola-Southwell coefficient for the $m$ th mode of vibration.

In a more general case, Equation (1a) may be written as a higher order polynomial, e.g. as a cubic function of $\Omega$,

$$
\omega_{m}^{2}(\Omega)=\omega_{m}^{2}(0) \pm s_{m} \Omega^{2} \pm r_{m} \Omega^{3} \pm \cdots
$$

Formula (1a) is widely attributed to Southwell but, according to Campbell (1924, p. 53), the formula was independently derived earlier by Stodola (1914). The formula derived by 
Southwell relates to uniform circular disks, while that of Stodola was derived for turbine blades. In any case, experience has shown that equation (1a) yields acceptable results not only for turbine blades and uniform circular disks, but also for other types of rotating structures such as helicopter blades, propellers, bladed disk assemblies, cylinders, etc.

\subsection{Intersection of Projected Eigenvalue Loci}

Consider a rotating blade modeled as a vibrating system having two degrees of freedom, such that there is no coupling between the two modes of vibration. The two uncoupled natural frequencies of the system may vary individually as functions of the rotation speed. For example, the second bending and first torsional frequencies of a rotating blade generally exhibit a variation with rotation speed. Let the natural frequencies at the non-rotating condition of the bending and torsion modes for such a blade be written as $\omega_{B}^{2}(0)$ and $\omega_{T}^{2}(0)$ respectively. The frequency loci of the bending mode $\omega_{B}(\Omega)$ and the torsion mode $\omega_{T}(\Omega)$ may appear as if they cross each other eventually at the rotation speed $\Omega^{*}$, as illustrated in Fig. 2 (a). Alternatively, the frequencies may diverge from each other, as illustrated in Fig. 2 (b). This follows from the fact that the Stodola-Southwell coefficients are not likely to be exactly equal for the two modes, so that the two frequency loci would not be curvilinearly "parallel".

As indicated earlier, there exist many studies in the literature on rotating blades where crossings of eigenvalue loci as in Fig. 2 (a) have been reported. However, although eigenvalue loci may apparently intersect, further analysis leads one to the conclusion that, in fact, such loci do not cross. The loci may approach each other arbitrarily closely, but they do not cross.

\subsection{Eigenvalue Loci of Rotating Blades Do Not Cross}

Observable frequency loci of rotating blades do not cross as a rule. There are at least two reasons why frequency loci cannot cross each other in a realistic model of rotating blades. The first follows from mathematical reasoning, and the other from physical or engineering considerations.

The mathematical reason why a crossing does not occur in the frequency loci of realistic models of rotating blades is due to the fact that such a crossing signifies degenerate eigenvalues (i.e coincident natural frequencies), and degenerate mathematical objects are "structurally unstable", according to a corollary of the weak transversality theorem (Thom \& Levin, 1959). As a result of the structural instability of degenerate objects, they are unobservable. Under the smallest perturbation, such degenerate and unobservable objects undergo a bifurcation and split into two or more structurally stable "generic" objects, which then become observable. This idea, called an "unfolding" by Thom, is very important in catastrophe theory, Thom (1972), the theory of singularities of differentiable mappings, 
Arnol'd et al. (1985), and the theory of algebraic curves, Brieskorn (1986).

The physical or engineering reason why a crossing of loci cannot be observed is due to the inevitable existence of a coupling between two or more modes in a vibrating system when such modes approach each other arbitrarily closely. This is called modal "interference", mode "coupling", or modal "interaction" in vibration analysis-see, for instance, Afolabi (1991) - and is evidenced by the existence of non-zero, off-diagonal terms in the system matrices. It is convenient to illustrate the foregoing with a two degree of freedom undamped system. If the two eigenvalues of such a system are equal (at the critical rotation speed at which the loci purportedly intersect), it would imply that the system has degenerate eigenvalues at that critical speed. However, the only way a coupled two degree of freedom system can have degenerate eigenvalues is when the system disintegrates into two identical, uncoupled sub-systems, so that $\omega_{1}=\omega_{2}$. But this would violate the assumption that the two degree of freedom system constitutes a coupled system, leading to a contradiction.

Although crossings of the eigenvalue loci of rotating blades do not occur in practice, such crossings are frequently reported in various publications in structural dynamics. In those theoretical studies where crossings have been reported, one finds that simplifying assumptions have usually been made (implicitly or otherwise) leading to a loss of modal coupling. In the case of experimental studies, what seems to be frequency crossings have been reported because, typically, it is very difficult or expensive to record the large number of data points that are required in the neighborhood of an assumed crossing, so that the experimenter is unable to resolve whether a crossing has actually taken place or not. If a special effort is made to gather sufficient data points in the neighborhood of the assumed crossing, then one may be able to resolve the presumed crossing into either an avoided crossing or a frequency coalescence.

\subsection{Coalescence of Eigenvalue Loci Induces Flutter}

In general, the quantitative difference between the eigenvalues in systems exhibiting a crossing, an avoided crossing or a frequency coalescence, is usually very small, except in the neighborhood of a crossing. Yet, for qualitative reasons, it is important to resolve an apparent crossing into a coalescence or veering. The importance of this lies in the fact that a frequency coalescence is a dangerous event, since it leads to flutter, whereas a curve veering does not usually lead to flutter and is, thus, relatively harmless.

If, in a flutter analysis, one mistakenly predicts a frequency coalescence condition as a frequency crossing or a curve veering, then evidently one has erroneously downgraded an unsafe flutter condition to a safe condition, with potential catastrophic consequences. Because there is a considerable qualitative difference between the dynamics of a system exhibiting frequency coalescence on the one hand, and a system characterized by curve veering or avoided crossing on the other hand, it is absolutely important to resolve an 
apparent crossing into either a frequency coalescence or a curve veering.

\section{Mode Coupling}

The existence or otherwise of mode coupling lies at the heart of a resolution of the question as to whether a frequency crossing does or does not occur in a realistic model of rotating blades. In what follows, we give three causes of mode coupling in rotating blades to support the view point that mode coupling should not be assumed to be completely absent.

\subsection{Mode Coupling Induced by Imperfection}

In theoretical analyses of an assembly of rotating blades (such as bladed disk assemblies), it is frequently assumed that all the blades (on a turbine disk, or in a helicopter rotor, for example) are completely identical. Such a model is called a "tuned" system. A theoretical model in which the effects of small amounts of imperfection or asymmetry are accounted for is called a "mistuned" model. However, it is impossible to manufacture all the blades in an assembly in such a way that they are all identical. Furthermore, small amounts of imperfection are also introduced into the system when the blades are mounted on the disk or hub. Moreover, wear and tear during normal operating conditions are inevitable, and these further introduce another level of imperfection. The probability that all these imperfections will somehow cancel one another out so that all the blades end up being exactly identical, is almost zero. Therefore, one must conclude that perfect geometric symmetry does not exist in a system of rotating blades. This lack of perfect geometric symmetry has serious physical consequences-it induces mode coupling, which could be conservative or non-conservative mode coupling.

It is easy to verify that curve veering may be induced by imperfection or "mistuning". First, one sets up a theoretical model accounting for mistuning (i.e imperfection, or geometric asymmetry such as static unbalance). Then the mistuning parameter is set to zero in one study, and to a non-zero value in another study, and the eigensolutions of the two studies subsequently compared.

For example, consider a simple lumped parameter model of a centrifugally loaded bladed disk with three blades on the disk, in which gyroscopic forces are ignored. Let $k_{c}$ be a nominal coupling stiffness, $k_{g}$ a "grounding" stiffness, and $\varepsilon$ a mistuning or imperfection

parameter of each blade. The following quasi-circulant matrix-form of the equations of motion of the mistuned cyclic system may be written,

$$
\ddot{\mathbf{x}}+\mathbf{K x}=0
$$


where

$$
\mathbf{x}^{T}=\left\{x_{1}, x_{2}, x_{3}\right\}
$$

and

$$
\mathbf{K}=\left[\begin{array}{ccc}
\left(k_{g}+2 k_{c}-\Omega^{2}\right) & -k_{c} & -k_{c} \\
-k_{c} & \left(k_{g}+2 k_{c}-\Omega^{2}\right) \pm \varepsilon & -k_{c} \\
-k_{c} & -k_{c} & \left(k_{g}+2 k_{c}-\Omega^{2}\right)
\end{array}\right]
$$

In the above, $x_{i}$ denotes the generalized coordinate of each blade. When the eigenvalues of the system (2) are plotted against the rotation speed, two loci belonging to two distinct modes of vibration would cross at $\Omega=0$ when $\varepsilon=0$. However, when the mistuning parameter is set to $\varepsilon \neq 0$, the loci do not cross at $\Omega=0$ but veer away from each other. The occurrence of curve veering is an evidence that a coupling of modes has taken place. This is analogous to what Poincaré calls the principle of "exchange of stabilities", and which may be called the principle of "exchange of modes" in our context. When only a small number of blades is involved (e.g. three or four, as in helicopters), it easier to appreciate the foregoing. In this regard, one may cite a recent study by Wang \& Chopra (1992).

\subsection{Mode Coupling Induced by Static Unbalance}

In addition to imperfection, mode coupling is also induced by static unbalance. In theoretical models of a rotating blade, for example, it is frequently assumed that the elastic

and geometric axes of a blade section coincide. In such an idealized case, there is no coupling whatsoever between the bending and torsional motions of the blade. Thus, a crossing of the bending and torsion eigenvalue loci may be reported from a study made using such an idealistic model.

In practical cases, however, an arbitrarily small amount of static unbalance of the crosssection is unavoidable during the manufacturing process. Thus, there always exists a small but non vanishing offset between the geometric and elastic axes, and this leads to a coupling of modes. Consequently, static unbalance in a rotating blade is another source of mode coupling.

\subsection{Mode Coupling Induced by Gyroscopic or Circulatory Forces}

Even if a single rotating blade had perfectly symmetric geometrical properties, or all traces of imperfections were somehow made to vanish completely in an assembly of blades, there exists yet another source of coupling one has to contend with, and that is from rotation and the aerodynamic environment.

Some coupling is introduced by gyroscopic forces, or other skew-symmetric forces induced by angular momentum. These are sometimes called "coriolis forces". Some coupling is also introduced by skew-symmetric forces induced by displacement, and these are some- 
times called "circulatory forces". Whereas gyroscopic coupling arises mainly from rotation, circulatory coupling originates mainly form aerodynamics. In some systems, both gyroscopic and circulatory coupling may be present at the same time; see, for instance, the book by Ziegler (1968).

\section{Conservative and Nonconservative Coupling}

A mathematical analysis of the vibration of a multi-degree of freedom system may be made in physical coordinates or in modal coordinates. The equations of motion are usually coupled in physical coordinates, and generally uncoupled in modal coordinates (for systems without damping, or with proportional damping). This is the case when the modes are not subject to parametrically dependent coupling. If the modal characteristics of a vibrating system depend on a parameter such as rotation speed, then although the equations of motion in modal coordinates may be uncoupled at a given rotation speed where the modes are well separated, there nevertheless will be a coupling of modes at another rotation speed when the modes become so close that they interact.

If, for example, one makes a two mode approximation of a rotating blade, one may assume that there is no parametric coupling whatsoever between the two modes. In that case, one may write the following equations of motion for vibrations, in the modal coordinates of bending and torsion for example, as

$$
\left[\begin{array}{ll}
1 & 0 \\
0 & 1
\end{array}\right]\left\{\begin{array}{l}
\ddot{\phi}_{B} \\
\ddot{\phi}_{T}
\end{array}\right\}+\left[\begin{array}{cc}
\omega_{B}^{2}(0)+s_{B} \Omega^{2} & 0 \\
0 & \omega_{T}^{2}(0)+s_{T} \Omega^{2}
\end{array}\right]\left\{\begin{array}{l}
\phi_{B} \\
\phi_{T}
\end{array}\right\}=0 .
$$

Without loss of generalization, unit modal mass has been assumed in the above. The eigenvalue matrix, $\mathrm{A}$, as in $\mathrm{Au}=\lambda \mathbf{u}$, of system (3) may be written as

$$
A=\left[\begin{array}{cc}
\omega_{B}^{2}(0)+s_{B} \Omega^{2}-\lambda & 0 \\
0 & \omega_{T}^{2}(0)+s_{T} \Omega^{2}-\lambda
\end{array}\right]
$$

where $\lambda=\omega^{2}(\Omega)$. Because of the assumption that there is no modal coupling in (3) or (4), the frequency loci are given by the equations

$$
\lambda_{1}=\omega_{1}^{2}(\Omega)=\omega_{B}^{2}(0)+s_{B} \Omega^{2} ; \quad \lambda_{2}=\omega_{2}^{2}(\Omega)=\omega_{T}^{2}(0)+s_{T} \Omega^{2} .
$$

These loci will either intersect or diverge, as in Figs. 2 (a) or 2 (b), respectively.

However, one may also assume that a parametric coupling does indeed exist between the bending and torsion modes. In that case, this coupling could be enforced in modal coordinates either as a conservative coupling, or as a non-conservative coupling; see, for instance, Crandall \& Mroszczyk (1988). 


\subsection{Conservative Coupling in Modal Coordinates}

If we consider a system without damping, then a coupling could take place between two modal coordinates, either in the modal mass matrix, or the modal stiffness matrix. Assuming a unit modal mass matrix, as before, and if we let $\beta \equiv \beta(\Omega)$ represent the coupling strength in the modal mass matrix while the modal stiffness matrix is uncoupled, one gets the following equations of motion in modal coordinates

$$
\left[\begin{array}{cc}
1 & -\beta \\
-\beta & 1
\end{array}\right]\left\{\begin{array}{l}
\ddot{\phi}_{B} \\
\ddot{\phi}_{T}
\end{array}\right\}+\left[\begin{array}{cc}
\omega_{B}^{2}(0)+s_{B} \Omega^{2} & 0 \\
0 & \omega_{T}^{2}(0)+s_{T} \Omega^{2}
\end{array}\right]\left\{\begin{array}{l}
\phi_{B} \\
\phi_{T}
\end{array}\right\}=0 .
$$

Alternatively, one may assume that the conservative coupling exists in the modal stiffness matrix but not in the modal mass matrix. In that case, the modal equations of motion may be written as

$$
\left[\begin{array}{ll}
1 & 0 \\
0 & 1
\end{array}\right]\left\{\begin{array}{l}
\ddot{\phi}_{B} \\
\ddot{\phi}_{T}
\end{array}\right\}+\left[\begin{array}{cc}
\omega_{B}^{2}(0)+s_{B} \Omega^{2} & -\beta \\
-\beta & \omega_{T}^{2}(0)+s_{T} \Omega^{2}
\end{array}\right]\left\{\begin{array}{l}
\phi_{B} \\
\phi_{T}
\end{array}\right\}=0 .
$$

In either case, one gets a linear algebraic eigenvalue problem, the eigenmatrix $\mathbf{A}$ of which is similarity invariant with the following matrix,

$$
\mathbf{A}=\left[\begin{array}{cc}
\omega_{B}^{2}(0)+s_{B} \Omega^{2}-\lambda & -\alpha \\
-\alpha & \omega_{T}^{2}(0)+s_{T} \Omega^{2}-\lambda
\end{array}\right]
$$

where $\alpha \equiv \alpha(\Omega), \beta \equiv \beta(\Omega)$. The eigenvalues of $A$ in (8) are

$$
\begin{aligned}
& \lambda_{1}=\omega_{1}^{2}(\Omega)=\frac{1}{2}\left[\omega_{B}^{2}(0)+\omega_{T}^{2}(0)+\left(s_{B}+s_{T}\right) \Omega^{2}\right]+ \\
& \sqrt{\alpha^{2}+\frac{1}{4}\left[\omega_{B}^{2}(0)-\omega_{T}^{2}(0)+\Omega^{2}\left(s_{B}-s_{T}\right)\right]^{2}} ; \\
& \lambda_{2}=\omega_{2}^{2}(\Omega)=\frac{1}{2}\left[\omega_{B}^{2}(0)+\omega_{T}^{2}(0)+\left(s_{B}+s_{T}\right) \Omega^{2}\right]- \\
& \sqrt{\alpha^{2}+\frac{1}{4}\left[\omega_{B}^{2}(0)-\omega_{T}^{2}(0)+\Omega^{2}\left(s_{B}-s_{T}\right)\right]^{2}} ;
\end{aligned}
$$

It may be noted that each of the two eigenvalues $\lambda_{1}, \lambda_{2}$ above, of the system (6) or (7) with conservative coupling, is a function of both the bending $\omega_{B}$ and torsion $\omega_{T}$ non-rotating frequencies, as well as the corresponding Stodola-Southwell coefficients for the bending and torsion modes, $s_{B}$ and $s_{T}$. If the conservative coupling vanishes completely, i.e $\alpha=0$, then (9) and (10) become, respectively,

$$
\lambda_{1}=\omega_{1}^{2}(\Omega)=\omega_{B}^{2}(0)+s_{B} \Omega^{2} ; \quad \lambda_{2}=\omega_{2}^{2}(\Omega)=\omega_{T}^{2}(0)+s_{T} \Omega^{2},
$$


which are the results previously obtained as (5) for the system without coupling. When the results of Equation (11) are plotted in the form of a frequency diagram, an intersection of loci results, as in Fig. 1 (b).

\subsection{Non-conservative Coupling in Modal Coordinates}

If the modal equations have non-conservative coupling in the modal mass matrix, one gets an equation of motion of the form

$$
\left[\begin{array}{cc}
1 & \beta \\
-\beta & 1
\end{array}\right]\left\{\begin{array}{l}
\ddot{\phi}_{B} \\
\ddot{\phi}_{T}
\end{array}\right\}+\left[\begin{array}{cc}
\omega_{B}^{2}(0)+s_{B} \Omega^{2} & 0 \\
0 & \omega_{T}^{2}(0)+s_{T} \Omega^{2}
\end{array}\right]\left\{\begin{array}{l}
\phi_{B} \\
\phi_{T}
\end{array}\right\}=0 .
$$

If, in the alternative, the non-conservative coupling exists in the modal stiffness matrix only, then the modal equations of motion may be written as

$$
\left[\begin{array}{ll}
1 & 0 \\
0 & 1
\end{array}\right]\left\{\begin{array}{l}
\ddot{\phi}_{B} \\
\ddot{\phi}_{T}
\end{array}\right\}+\left[\begin{array}{cc}
\omega_{B}^{2}(0)+s_{B} \Omega^{2} & \beta \\
-\beta & \omega_{T}^{2}(0)+s_{T} \Omega^{2}
\end{array}\right]\left\{\begin{array}{l}
\phi_{B} \\
\phi_{T}
\end{array}\right\}=0 .
$$

The eigenvalue problem of (12) or (13) has an eigenmatrix $\mathbf{A}$ which is similar to the following matrix,

$$
\mathbf{A}=\left[\begin{array}{cc}
\omega_{B}^{2}(0)+s_{B} \Omega^{2}-\lambda & \alpha \\
-\alpha & \omega_{T}^{2}(0)+s_{T} \Omega^{2}-\lambda
\end{array}\right]
$$

The eigenvalues of the above are

$$
\begin{aligned}
& \lambda_{1}=\omega_{1}^{2}(\Omega)=\frac{1}{2}\left[\omega_{B}^{2}(0)+\omega_{T}^{2}(0)+\left(s_{B}+s_{T}\right) \Omega^{2}\right]+ \\
& \sqrt{-\alpha^{2}+\frac{1}{4}\left[\omega_{B}^{2}(0)-\omega_{T}^{2}(0)+\Omega^{2}\left(s_{B}-s_{T}\right)\right]^{2}} ; \\
& \lambda_{2}=\omega_{2}^{2}(\Omega)=\frac{1}{2}\left[\omega_{B}^{2}(0)+\omega_{T}^{2}(0)+\left(s_{B}+s_{T}\right) \Omega^{2}\right]- \\
& \sqrt{-\alpha^{2}+\frac{1}{4}\left[\omega_{B}^{2}(0)-\omega_{T}^{2}(0)+\Omega^{2}\left(s_{B}-s_{T}\right)\right]^{2}}
\end{aligned}
$$

The relative signs of the off-diagonal terms in (14) determine whether the coupling is conservative or non-conservative. A symmetric stiffness coupling is conservative, whereas a skew-symmetric coupling is non-conservative.

\section{Composition of Plane Algebraic Curves}

Most of the mathematical ideas and techniques currently used in engineering analysis 
date back to three or more centuries. Newer results arising from recent research in mathematics (from the 1950 s to the present, for instance), are hardly utilized in engineering analysis at present, mainly due to the inaccessible language utilized by mathematicians. According to V. I. Arnol'd (1989, p. ix),

"The new discoveries ... have potentially extremely wide applications, but since these results were discovered rather recently, they are discussed only in specialized [mathematical journals], and applications are impeded by the difficulty of the mathematical exposition for the nonmathematicians."

In the course of our discussion in this paper, we shall find it useful to apply some relatively new results and ideas from algebraic geometry: the weak transversality theorem from catastrophe theory (Thom \& Levin, 1959; Thom, 1972), and the effect of small perturbations on the composition of real algebraic curves, Brieskorn (1986).

The transversality theorems (the weak transversality theorem and Thom's transversality theorem) are very useful in studying the qualitative behavior of mathematical objects in the neighborhood of a degeneracy. In recent years, these theorems have been used directly, or indirectly through catastrophe theoretic formalisms, by Afolabi (1989, 1991, 1993a, 1993b), to furnish insight into the qualitative behavior and "structural stability" of linear vibrating systems.

The effect of small perturbations on the composition of real algebraic curves has been discussed by Arnol'd (1983) and Brieskorn (1986), among others. In demonstrating that the observable eigenvalue loci belonging to two distinct modes do not intersect, we shall find it useful to study the local topology of real algebraic curves in the plane, in the neighborhood of a presumed "intersection".

\subsection{Linear Characteristics of Smooth Functions}

Thom's classification theorem of catastrophe theory is based on the differentiable topology of smooth functions in one or two variables, and their (uni)versal unfoldings. By a "smooth function" is meant an infinitely differentiable function, also called a $C^{\infty}$ function. The locus of a given eigenvalue of a rotating blade as a function of the rotation speed is a $C^{\infty}$ function. It follows from the Implicit Function Theorem that any $C^{\infty}$ function may be linearized everywhere, except in the neighborhood of its critical points. In general, the eigenvalue loci of a rotating blade, or a system of rotating blades, do not have critical points (except, possibly, at the non-rotating frequency). Therefore, the eigenvalue loci may be approximated as linear functions at almost all rotation speeds.

Consider the neighborhood of a point where two eigenvalue loci are presumed to intersect. We now propose to show that given an arbitrarily small amount of perturbation (or, a coupling of the two curves), the "intersection" will disappear, leaving either an avoided crossing or a frequency coalescence. 


\subsection{Effect of Coupling on the Composition of Plane Algebraic Curves}

Suppose, in the neighborhood of a "crossing", that the two curves that are presumed to intersect have linear representations, as a consequence of the Implicit Function Theorem. Then, using the illustration in Fig. 1 (a) as an example, the two curves may be described as polynomials of the first degree. Consequently, they are real algebraic curves in the plane. Thus, we may write

$$
p_{1} \equiv y=m_{1} x+c_{1}, \quad p_{2} \equiv y=m_{2} x+c_{2},
$$

where $m_{i}$ is the slope of the $i$ th curve, and $c_{i}$ its intercept with the $y$-axis.

From the local algebra of plane algebraic curves, Arnol'd (1983, p. 78), or Brieskorn (1986) the composite curve may be written as

$$
p \equiv p_{1} \circ p_{2}=0,
$$

which corresponds, in this case, to an ordinary multiplication of the two polynomials, i.e.

$$
\left(y-m_{1} x-c_{1}\right) \cdot\left(y-m_{2} x-c_{2}\right)=0 .
$$

However, the procedure of composition of algebraic curves is a mathematical "surgery", in which the neighborhood of the intersection is deleted and the resulting pieces "glued" together. All this is done mathematically, using standard techniques of algebraic topology; see, for instance, the review article by Atiyah (1975). As a result of this "surgery", the right hand side of (19) is not completely zero, but a small, non-vanishing real number, here denoted by $\varepsilon$. Thus, although (19) is theoretically imaginable, it is not practically realizable, and (19) therefore becomes

$$
\left(y-m_{1} x-c_{1}\right) \cdot\left(y-m_{2} x-c_{2}\right)=\varepsilon .
$$

Expanding the above yields

$$
y^{2}-\left[\left(m_{1}+m_{2}\right) x+\left(c_{1}+c_{2}\right)\right] y+\left[\left(c_{1} c_{2}-\varepsilon\right)+\left(m_{1} c_{2}+m_{2} c_{1}\right) x+m_{1} m_{2} x^{2}\right],
$$

which may be solved as a quadratic in $y$ to get

$$
y(x ; \varepsilon)=\frac{\left(m_{1}+m_{2}\right) x+\left(c_{1}+c_{2}\right) \pm \sqrt{\left[\left(m_{1}-m_{2}\right) x+\left(c_{1}-c_{2}\right)\right]^{2}+4 \varepsilon}}{2} .
$$

Equation (22) is a general form from which one could generate composite curves having qualitatively different differentiable topological characteristics. For example, one could generate the three qualitatively different curves or "loci" in Figs. 1 by using the following 
parameters, $m_{1}=1, m_{2}=-1, c_{1}=1, c_{2}=2$ to get the parametric equation

$$
(y-x-1)(y+x-2)-\varepsilon=0, \quad \Rightarrow y^{2}-3 y-\left(x^{2}-x-2+\varepsilon\right)=0,
$$

which, when solved, yields

$$
y(x ; \varepsilon)=1.5 \pm \sqrt{(x-0.5)^{2}+\varepsilon}
$$

The degenerate case of (19) is obtained when the parameter $\varepsilon=0$, yielding the intersecting loci in Fig. 1 (a). In this case, the effect of "perturbation" on the surgical procedure involved in the composition of the two curves is assumed to have vanished completely. If, however, we set $\varepsilon>0$, i.e. to any arbitrary positive real number, then the curves in Fig. 1 (b) result. This is similar to the case of "curve veering" well known in structural dynamics. Larger values of $\varepsilon$ give rise to stronger coupling, while smaller values correspond to weaker coupling. As $\varepsilon \rightarrow 0$, the two curves develop very sharp local curvatures. They approach each other arbitrarily closely, and may appear to cross each other when $\varepsilon$ is almost zero. However, provided $\varepsilon$ does not exactly vanish, the two composing curves do not cross each other. If, on the other hand, we set $\varepsilon<0$, i.e. to any arbitrary negative real number, then we get the curves shown in Fig. 1 (c). This is similar to the case of "frequency coalescence" characteristic of coupled mode flutter, which is well known in aeroelasticity. The separation between the two segments of the now surgically coupled curves indicate a flutter zone. At any rotation speed within this zone, the rotating blade flutters, and becomes unstable. As in the preceding case of curve veering, smaller values of $\varepsilon$ imply weaker coupling, while large values imply strong coupling. The stronger the coupling, the larger the flutter zone.

Similar results to Figs. 1 may be obtained by using different values of $m_{i}, c_{i}$, and $\varepsilon$ in (20). For example, one may set $m_{1}=1, m_{2}=0, c_{1}=1, c_{2}=2$, and $\varepsilon=0, \varepsilon>0, \varepsilon<0$ for the (a), (b) and (c) versions of Figs. 3. Globally, the eigenvalue loci as functions of rotation speed may be illustrated as in Figs. 4.

From what has been said above, we come to the conclusion that a composition of two plane curves yields a crossing if and only if $\varepsilon=0$. Such an intersection is, however, not in "general position", and an infinitesimally small amount of perturbation, signified by $\varepsilon \neq 0$, will induce a topological surgery which manifests either as "curve veering" or "frequency coalescence".

\section{Physical Significance of Curve Veering and Frequency Coalescence}

Fig. 3 (a) shows two eigenvalue loci which intersect; the two loci in Fig. 3 (b) repel each other, exhibiting the curve veering phenomenon; while Fig. 3 (c) is characterized by the coalescence of the two loci. 
If we superimpose the curves in Figs. $3(\mathrm{a}),(\mathrm{b})$ and (c), the quantitative difference between the eigenvalue loci in a given diagram and the corresponding loci in the other two diagrams is very small. The largest difference occurs near the point where the uncoupled curves intersect in Fig. 3 (a). Yet, the dynamics represented by the three diagrams are significantly different from one another in a qualitative sense. In this section, we discuss the importance of frequency coalescence on the one hand, and curve veering on the other, with regard to the flutter and stability of a rotating blade or a rotating system of blades.

\subsection{Frequency Crossing Implies Eigenvalue Degeneracy}

Two loci can only cross each other if and only if there is zero coupling between the vibration modes represented by each eigenvalue locus. For example, the eigenlocus of the torsion branch will cross that of the bending branch only if there is no coupling whatsoever between the bending and torsion modes. If such a crossing of eigenloci were to occur in an actual engineering system, then the system's eigenvalues must be degenerate at the point where the two loci meet.

However, the occurrence of degenerate eigenvalues in a coupled two degree of freedom vibrating system is impossible, from physical or engineering considerations, as discussed in $\S 2.3$. Thus, the type of eigenvalue loci shown in Fig. 3 (a) cannot occur in a coupled system with two degrees of freedom (e.g. bending and torsion degrees of freedom).

Theoretically, one could always assume, of course, that a coupled two degree of freedom model with degenerate eigenvalues existed, in which case the loci would intersect. The degenerate eigenvalues at each intersection point could also be assumed to have a linearly independent set of eigenvectors, so that the eigenvalue and eigenvector matrices may be written, respectively, as

$$
\Lambda=\left[\begin{array}{ll}
\lambda & 0 \\
0 & \lambda
\end{array}\right], \quad \mathrm{U}=\left[\begin{array}{ll}
1 & 0 \\
0 & 1
\end{array}\right],
$$

at the intersection point.

In such a case, the physically unrealizable system having the above eigensolution would have "elastic stability" (since the eigenvectors are linearly independent), but not "structural stability" in the topological sense (because the eigenvalues are degenerate).

\subsection{Frequency Coalescence Implies Eigenvector Degeneracy and, Consequently, Flutter}

Consider now, the case depicted in Fig. 3 (c). Each of the two points at which the loci belonging to two distinct modes coalesce constitutes a flutter boundary. To see this, one observes that the eigenvalue matrix of the system may be written in a canonical form due 
to Crandall \& Mroszczyk (1988) as

$$
A=\left[\begin{array}{cc}
1-\alpha & -\beta \\
\beta & 1+\alpha
\end{array}\right]
$$

The eigenvalues, as well as the eigenvectors, of (26) are degenerate when $\alpha= \pm \beta$, which are the two flutter boundaries. The implication of eigenvector degeneracy in this case is very different from that of eigenvalue degeneracy considered in $\$ 6.1$. An important fact for system dynamics in this case is this: at the flutter boundaries, because the eigenvectors are also degenerate, the system matrix cannot be diagonalized; it is only reducible to the Jordan normal form. Thus, the eigensolution at the flutter boundaries may be written in the Jordan canonical form

$$
\Lambda=\left[\begin{array}{cc}
\lambda & 0 \\
1 & \lambda
\end{array}\right], \quad \mathrm{U}=\left[\begin{array}{ll}
1 & 1 \\
0 & 0
\end{array}\right], \quad \mathrm{V}=\left[\begin{array}{cc}
1 & -1 \\
0 & -1 / \alpha
\end{array}\right],
$$

where $\mathbf{U}$ is a matrix of ordinary eigenvectors, while $\mathrm{V}$ is a matrix of generalized eigenvectors of the system matrix. From the foregoing, one observes that the eigenvalue matrix is not diagonal, and the matrix of ordinary eigenvectors is singular. Due to the singularity, or noninvertibility, of the ordinary eigenvector matrix, $U$, a matrix of generalized eigenvectors, $\mathrm{V}$, must be computed. By means of these generalized eigenvectors a non-singular linear change of coordinates may be found so that the system matrix may be reduced to the Jordan normal form. It is easy to show that the existence of a Jordan matrix in a linear vibrating system necessarily implies the occurrence of flutter in that system.

\subsection{Curve Veering Does Not Lead to Flutter}

In order for flutter to occur in an undamped vibrating system with two degrees of freedom, the two eigenvalues of the system must coalesce at the flutter boundary, leading to eigenvalue degeneracy. More significantly, the corresponding eigenvectors must also be degenerate, so that the system matrix becomes non-diagonalizable at the flutter boundary. Such a situation cannot arise in an undamped system which exhibits curve veering, for the simple reason that the two eigenvalues repel each other when they get arbitrarily close, thereby preventing a degeneracy or mode coalescence. The system exhibiting curve veering may, nevertheless, lose elastic stability if net negative damping is later added, and it may lose elastic stability by "divergence", when one of its eigenvalues becomes zero.

\section{Effect of Damping on Flutter Boundaries}

The discussion in the preceding sections of this paper has been based on a model without 
damping. In this section, we briefly discuss the role of damping on the systems exhibiting curve veering on the one hand, and frequency coalescence on the other.

As discussed earlier, systems which give rise to curve veering only cannot flutter when there is no damping. Introducing positive damping to the system is generally beneficial, and does not usually destabilize the system.

Undamped systems giving rise to frequency coalescence can behave in very interesting ways when positive damping is added to the system. In the cases where mode coalescence is due to circulatory forces (i.e. skew-symmetric stiffness coupling, which is the same as nonconservative coupling), the introduction of positive damping to the system has a beneficial effect-the flutter zone is reduced. On the other hand, where the coalescence is due to gyroscopic forces, (i.e. skew-symmetric coupling via the damping matrix), the addition of positive damping worsens the situation, and further destabilizes the system. Thus, the flutter zone is widened by damping, so that flutter occurs at a lower rotation speed relative to the corresponding undamped system. In either case (of circulatory or gyroscopic coupling), damping may prevent the two vibration frequencies from coalescing. Therefore, a major difference between a damped and an undamped model of a fluttering system is that the eigenvalue loci actually coalesce at the flutter boundary when there is no damping, but they do not necessarily coalesce at the flutter boundary when damping is added to the system.

\section{CONCLUSIONS}

In this paper, some standard results from the theory of plane algebraic curves and from catastrophe theory have been used to show that the loci of two or more eigenvalues depending on a common parameter (such as rotation speed, $\Omega$ ) almost always do not cross. Cases where such eigenvalue loci are reported to have crossed exist only in unrealistic theoretical models where it is assumed that no coupling exists between the two different modes of vibration. If, in an experimental work insufficient data are recorded due to a variety of practical factors, it is quite possible to erroneously interpolate the experimental data in such a way as to imply that the loci cross each other. In reality, however, frequency loci of undamped rotating blades do not cross, but must either repel each other leading to curve veering, or attract each other leading to frequency coalescence.

From the mathematical point of view, a crossing of eigenvalue loci implies the existence of degenerate eigenvalues. Degenerate objects of a category are not in "general position", and are necessarily "structurally unstable". Therefore, they are unobservable. The existence of degenerate eigenvalues also indicates a non-transversal condition. By the weak transversality theorem, an arbitrarily small shift will lead to a bifurcation of a non-transversal

intersection leading to algebraic curves exhibiting veering or coalescence which will then be in general position and are thus "structurally stable". 
From the engineering point of view, a crossing of eigenvalue loci implies the existence of degenerate eigenvalues, such that the eigenvectors are independent at the degeneracy. Two or more eigenvalue loci of a rotating blade intersect only in theoretical studies where it is presumed that some form of perfect symmetry exists, and there is zero coupling of modes. Minute asymmetry or imperfections are inevitable in practice, and these lead to a coupling of neighboring modes. If the coupling is conservative, then the eigenvalue loci would exhibit avoided crossings or curve veering. On the other hand, if there is non-conservative coupling, the eigenvalue loci exhibit frequency coalescence.

Frequency coalescence in an undamped rotating blade, or system of blades, is very dangerous since it leads to flutter. On the contrary, curve veering in an undamped rotating blade or system of rotating blades is benign with respect to flutter.

\section{ACKNOWLEDGEMENT}

Part of the work of the first author was supported by a grant at ICOMP, NASA Lewis Research Center, Cleveland, Ohio. The support is gratefully acknowledged.

\section{REFERENCES}

1. Afolabi, D. 1989 Effects of Mistuning and Matrix Structure on the Topology of Frequency Response Curves. NASA TM 102290.

2. Afolabi, D. 1991 Modal Interaction in Linear Dynamic Systems Near Degenerate Modes. NASA TM 105315.

3. Afolabi, D. 1993a "Flutter analysis using transversality theory" (forthcoming in Acta Mechanica).

4. Afolabi, D. 1993b "The cusp catastrophe and the stability problem of helicopter ground resonance". Proc. R. Soc. Lond., A441, 399-406.

5. Andronov, A. A. and Vitt, A. A. 1966 Theory of Oscillators London: Pergamon. (Republished 1987 at New York: Dover).

6. Arnol'd. V. I. 1983 Catastrophe Theory. New York: Springer-Verlag.

7. Arnol'd, V. I., Gusein-Zade, S. M. and Varchenko, A. N., 1985, Singularities of Differentiable Maps, Vol. 1. B̈̈rkhauser, Boston, MA.

8. Arnol'd. V. I. 1989 Mathematical Methods of Classical Mechanics, 2nd. Ed. New York: Springer-Verlag. 
9. Atiyah, M. F. 1976 "Bakerian Lecture, 1975-Global geometry". Proc. R. Soc. Lond., A347, 291-299.

10. Bisplinghoff, R. L. and Ashley, H. 1962 Principles of Aeroelasticity. New York: Dover Publications.

11. Brieskorn, E. 1986 Plane Algebraic Curves. Boston: Birkhauser.

12. Campbell, W. 1924 "The protection of steam turbine disk wheels from axial vibration". Trans. ASME, 46, 31-160.

13. Chen, L.-T. and Dugundji, J. 1984 "Investigation of the vibration characteristics of shrouded bladed disk rotor stages". J. Aircraft, 17, 479-486.

14. Crandall, S. H., and Mroszczyk, J. W. 1988 "Conservative and nonconservative coupling in dynamic systems'. Proc. Intl. Conf. Vib. Rotating Mach., Cambridge, 567-572. London: IMechE.

15. Lamb, H. and Southwell, R. V. 1921 "The vibrations of a spinning disc". Proc. $R$. Soc. Lond., A99, 272-280.

16. Leissa, A. W. 1974 "On a curve veering aberration". ZAMP (J. appl. Math. Phys.), 25, pp. 99-111.

17. MacBain, J. 1975 "Vibratory behavior of twisted cantilevered plates". J. Aircraft, 12, 343-349.

18. Perkins, N. C. and Mote, C. D. 1988 "Comments on curve veering in eigenvalue problems". J. Sound Vib., 106, 451-463.

19. Poincaré, H. 1892 New Methods of Celestial Mechanics. NASA TT-F-450, 451, 452. National Technical Information Service, Springfield, VA. (In French: Les Méthodes Nouvelle de la Mécanique Céleste, Dover, New York, 1957; Guthier-Villas, Paris, $1892,1893,1899)$.

20. Ramamurti, V. and Kielb, R. E. 1984 "Natural frequencies of twisted rotating plates". J. Sound Vib., 97, 429-449.

21. Southwell, R. V., 1922 "On the free transverse vibrations of a uniform circular disc clamped at its centre and on the effect of rotation". Proc. R. Soc. Lond., A101, 133-153.

22. Stodola, A. 1914 Schweitzerische Bauzeitung, May 1914. 
23. Thom, R. and Levin, H. I. 1959 "Singularities of Differentiable Mappings", Bonn Math. Schri. (Reprinted in Lecture Notes in Math. \#192, New York: Springer, 1971).

24. Thom, R. 1972 Structural Stability and Morphogenesis, Reading, MA: Benjamin. (Republished by Addison Wesley, Reading, MA, 1989).

25. Wang, J. M. and Chopra, I. 1992 "Dynamics of helicopters in ground resonance with and without blade dissimilarities". AIAA Paper 92-.

26. Ziegler, H. 1968 Principles of Structural Stability Waltham, Mass.: Blaisdell. 
(a)

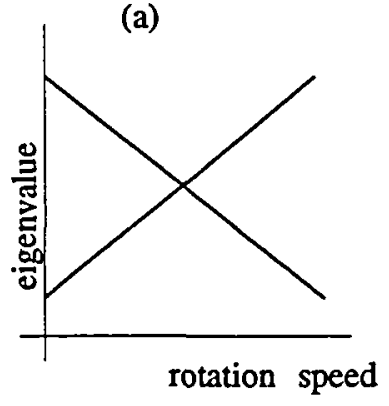

(b)

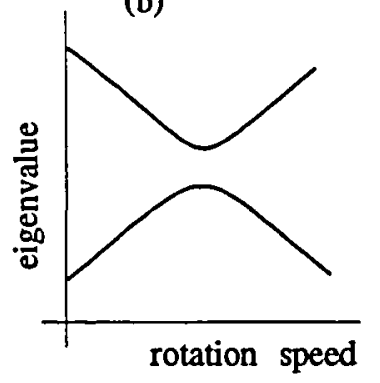

(c)

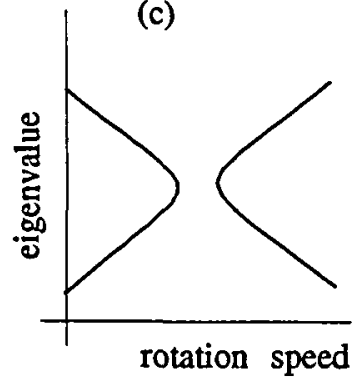

Fig. 1: (a) Frequency crossing; (b) curve veering; and (c) frequency coalescence.
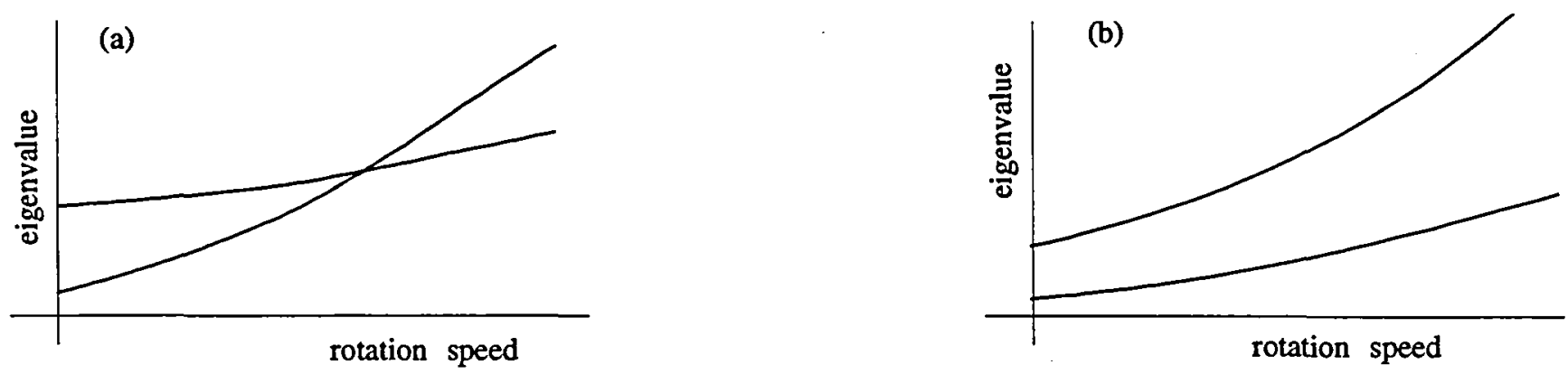

Fig. 2: Variation of resonance frequencies with rotation speed showing (a) intersecting loci; (b) diverging loci.
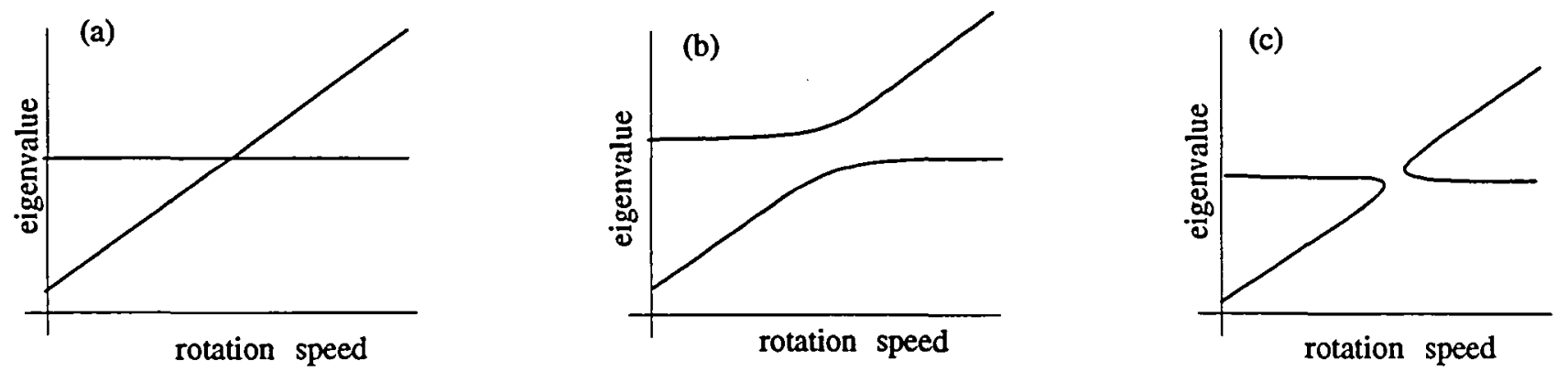

Fig. 3: Two loci, one showing linear variation of resonance frequency with rotation speed, the other invariant with speed; (a) frequency crossing; (b) curve veering; (c) frequency colescence.
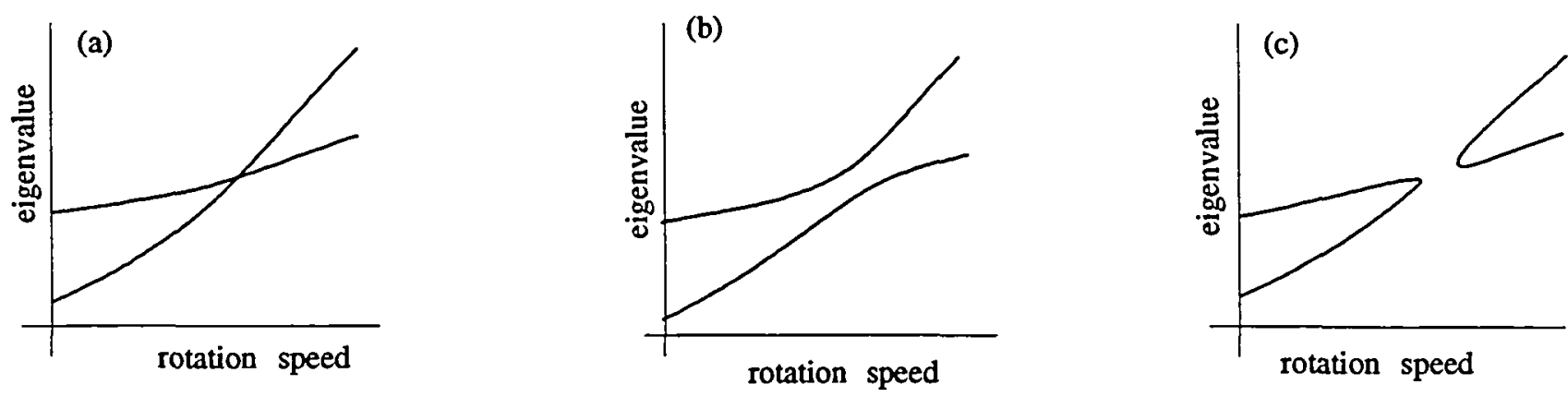

Fig. 4: Nonlinear variation of resonance frequencies with rotatiuon speed showing (a) frequency crossing; (b) curve veering; (c) frequency colescence. 
1

, 
Public reporting burden for this collection of information is estimated to average 1 hour per response, including the time for reviewing instructions, searching existing data sources, gathering and maintaining the data needed, and completing and reviewing the collection of information. Send comments regarding this burden estimate or any other aspect of this collection of information, including suggestions for reducing this burden, to Washington Headquarters Services, Directorate for Information Operations and Reports, 1215 Jefferson Davis Highway. Suite 1204, Arlington, VA 22202-4302, and to the Office of Management and Bucget, Paperwork Reduction Project (0704-0188), Washington, DC 20503.

\begin{tabular}{|l|c|c|}
\hline 1. AGENCY USE ONLY (Leave blank) & $\begin{array}{c}\text { 2. REPORT DATE } \\
\text { August } 1993\end{array}$ & $\begin{array}{r}\text { 3. REPORT TYPE AND DATES COVEAED } \\
\text { Technical Memorandum }\end{array}$ \\
\hline
\end{tabular}

\section{TITLE AND SUBTTLE}

On Curve Veering and Flutter of Rotating Blades

5. FUNDING NUMBERS

Daré Afolabi and Oral Mehmed

WU-505-90-5K

6. AUTHOR(S)
Daré Afolabi and Oral Mehmed

\section{PERFORMING ORGANIZATION NAME(S) AND ADDRESS(ES)}

National Aeronautics and Space Administration

Lewis Research Center

Cleveland, Ohio 44135-3191

8. PERFORMING ORGANIZATION REPORT NUMBER

E-8020

\section{SPONSORING/MONTORING AGENCY NAME(S) AND ADDRESS(ES)}

National Aeronautics and Space Administration

Washington, D.C. 20546-0001
10. SPONSORING/MONTORING AGENCY REPORT NUMBER

NASA TM-106282

ICOMP-93-27

11. SUPPLEMENTARY NOTES

Daré Afolabi, Institute for Computational Mechanics in Propulsion, NASA Lewis Research Center and Purdue University, Department of Mechanical Engineering, Indianapolis, Indiana 47907 (work funded under NASA Cooperative Agreement NCC3-233) and Oral Mehmed, NASA Lewis Research Center. ICOMP Program Director, Louis A. Povinelli, (216) 433-5818.

12a. DISTRIBUTION/AVAILABILTY STATEMENT

12b. DISTRIBUTION CODE

Unclassified - Unlimited

Subject Category 39

\section{ABSTAACT (Maximum 200 words)}

The eigenvalues of rotating blades usually change with rotation speed according to the Stodola-Southwell criterion. Under certain circumstances, the loci of eigenvalues belonging to two distinct modes of vibration approach each other very closely, and it may appear as if the loci cross each other. However, our study indicates that the observable frequency loci of an undamped rotating blade do not cross, but must either repel each other (leading to "curve veering"), or attract each other (leading to "frequency coalescence"). Our results are reached by using standard arguments from algebraic geometry - the theory of algebraic curves and catastrophe theory. We conclude that it is important to resolve an apparent crossing of eigenvalue loci into either a frequency coalescence or a curve veering, because frequency coalescence is dangerous since it leads to flutter, whereas curve veering does not precipitate flutter and is, therefore, harmless with respect to elastic stability.

14. SUBJECT TERMS

Curve veering; Eigenvalue loci; Flutter; Frequency crossing 21

16. PRICE CODE

$\mathrm{AO3}$

\begin{tabular}{|c|c|c|}
\hline $\begin{array}{c}\text { 17. SECURITY CLASSIFICATION } \\
\text { OF REPORT } \\
\text { Unclassified }\end{array}$ & $\begin{array}{c}\text { 18. SECURITY CLASSIFICATION } \\
\text { OF THIS PAGE } \\
\text { Unclassified }\end{array}$ & $\begin{array}{c}\text { 19. SECURTY CLASSIFICATION } \\
\text { OF ABSTRACT } \\
\text { Unclassified }\end{array}$ \\
\hline
\end{tabular}


National Aeronautics and Space Administration

Lewis Research Center ICOMP (M.S. 5-3)

Cleveland, Ohio 44135

OHicial Buelneas

Penatiy for Private Uee $\$ 300$
FOURTH CLASS MAIL.

\section{ADDRESS CORRECTION REQUESTED}

